\title{
The 3D Visualization of Brain Anatomy from Diffusion-Weighted Magnetic Resonance Imaging Data
}

\author{
Burkhard C. Wünsche* and Richard Lobb ${ }^{\dagger}$ \\ Division for Biomedical Imaging and Visualization, Department of Computer Science \\ University of Auckland, Auckland, New Zealand
}

\begin{abstract}
A common problem in biomedical sciences is the in vivo identification and analysis of anatomical structures. This paper introduces several novel techniques to identify and visualize nerve fiber tracts and different tissue types using diffusion-weighted magnetic resonance imaging data. Barycentric color maps allow an integrated view of different types of diffusion anisotropy. Ellipsoid-based textures and Anisotropy Modulated Line Integral Convolution create images segmented by tissue type and incorporating a texture representing the $3 \mathrm{D}$ orientation of nerve fibers. Finally streamtubes and hyperstreamlines represent the full 3D structure of nerve fiber tracts and their inherent diffusion properties. The effectiveness of the exploration approach and the new visualization techniques are demonstrated by identifying various anatomical structures and features from a diffusion tensor data set of a healthy brain.
\end{abstract}

CR Categories: I.3.8 [Computer Graphics]: Applications; I.3.6 [Computer Graphics]: Methodology and Techniques-Graphics Data Structures and data types; J.3 [Computer Applications]: Life and Medical Sciences

Keywords: Biomedical Visualization, Tensor Field Visualization, Diffusion Tensor Imaging, Brain Anatomy, Nerve Fiber Tracking

\section{Introduction}

Medical imaging is an essential tool for improving the diagnoses, understanding and treatment of a large variety of diseases. This paper presents techniques for obtaining in vivo information of the brain anatomy by visualizing and systematically exploring Diffusion Tensor Imaging (DTI) data. The diffusion tensor describes the spatial distribution of water molecules originating at a common location. Since the diffusion of water depends on the micro-structure of the tissue, the diffusion tensor field can be used to visualize nerve fibers and other tissues in the brain. The resulting images of the brain anatomy can be used to advance research in surgical planning, cognitive sciences, and the diagnosis and treatment of various white and gray matter disorders [Pierpaoli et al. 1996; Lim et al.

*e-mail: burkhard@cs.auckland.ac.nz

†e-mail: richard@cs.auckland.ac.nz
1999; Barnea-Goraly et al. 2003]. Visualizing the nerve fiber structure also represents a valuable teaching tool.

Our approach starts with slice images familiar to the medical specialist and progressively expands the dimension and abstraction level of the representation in order to provide new insights into the data. In particular we present four innovative techniques for the visualization of DTI data. Barycentric color maps allow an integrated view of different types of diffusion anisotropy. Ellipsoid-based textures in combination with spherical or barycentric color maps indicate the nerve fiber direction and different anisotropy properties and tissue types. Anisotropy Modulated Line Integral Convolution (AMLIC) creates images segmented by tissue type and incorporating a texture representing the $3 \mathrm{D}$ orientation of nerve fibers. Finally streamtubes and hyperstreamlines visualize the complex 3D structure of nerve fiber tracts and their inherent diffusion properties.

All images in this paper were created with a toolkit we developed specifically for visualizing biomedical data sets [Wünsche 2003b].

\section{Diffusion Tensor Imaging}

Diffusion tensor imaging (DTI), also known as diffusion-weighted MRI imaging (DWI), is used to measure the intrinsic properties of water diffusion in the brain by an orientation invariant quantity, the diffusion tensor D [Basser et al. 1994; Basser 1995]. The eigenvalues and eigenvectors of the symmetric second-order tensor $\mathbf{D}$ define the principal axes of a diffusion ellipsoid which expresses the spatial distribution of water molecules originating at a point location after an infinitesimal time period.

DTI almost completely suppresses effects due to water in the blood vessels [Basser 2000] and can be used to measure the diffusion of cerebral spinal fluid (CSF) and fluid inside of nerve cells. The results of the measurement are the six components of the symmetric diffusion tensor $\mathbf{D}$ and the $T_{2}$ weighted signal intensity in the absence of diffusion sensitization. Images of water diffusion can provide pathophysiological information complementary to $T_{1}$ and $T_{2}$ weighted MRI images. The technique is sensitive to movements of the order of a few microns and is described in more detail in [Basser and Pierpaoli 1996; Pierpaoli et al. 1996; Aronen et al. 1999].

In the brain DTI can be used to differentiate three types of structures. Fluid filled compartments are characterized by a very high isotropic diffusion, i.e., the diffusion is similar in all directions. In contrast nerve fibers restrict the diffusion to one direction only due to the presence of cell membranes and myelin sheaths surrounding the axons. Fiber tracts, consisting of millions of parallel nerve fibers, are therefore identified as areas of a high anisotropic diffusion. The orientation of such fiber tracts is determined from the principal directions of the diffusion tensor. Finally gray matter is characterized by a low and nearly isotropic diffusion since the water diffusion is restricted in all directions due to cell membranes of intermingled cell bodies and their surrounding neuraglia. Consequently DTI can be used to gain in vivo information about the anatomy, microstructure and physiology of the brain. 


\subsection{Derived Quantities}

The matrix representation of a second-order tensor depends on the coordinate system used (MRI coordinates). In order to describe intrinsic tissue properties variables independent of the patient's position must be derived. Examples are the three tensor invariants. An important property of any 3-dimensional symmetric second-order tensor $\mathbf{T}$ is that there always exist 3 eigenvalues $\lambda_{i}$ and 3 mutually perpendicular eigenvectors $\mathbf{v}_{i}$ such that

$$
\mathbf{T} \mathbf{v}_{i}=\lambda_{i} \mathbf{v}_{i} \quad i=1, \ldots, 3
$$

The eigenvalues and eigenvectors of the diffusion tensor $\mathbf{D}$ define the principal axes of a diffusion ellipsoid. To facilitate the definition of new measures it is convenient to order the three eigenvalues of the diffusion tensor $\mathbf{D}$ by size with $\lambda_{1}$ being the biggest and $\lambda_{3}$ being the smallest [Pierpaoli et al. 1996]. The maximum diffusivity is then given by $\lambda_{\max }=\lambda_{1}$.

The mean diffusivity is defined as the average eigenvalue of the diffusion tensor and is efficiently computed by using the first tensor invariant

$$
\lambda_{\text {mean }}=\frac{\lambda_{1}+\lambda_{2}+\lambda_{3}}{3}=\frac{\operatorname{trace}(\mathbf{D})}{3}=\frac{D_{11}+D_{22}+D_{33}}{3}
$$

Images of $\lambda_{\text {mean }}$ show all brain tissue and fluid filled compartments. Note that the computation does not require the computation of the eigenvalues but involves merely averaging the diagonal elements of the tensor matrix.

Another important measure is the anisotropy of the diffusion tensor. We define it as

$$
\begin{aligned}
\lambda_{\text {aniso }} & =\operatorname{trace}\left(\left(\mathbf{D}-\lambda_{\text {mean }} \mathbf{I}\right)^{2}\right) / \lambda_{\text {mean }}^{2} \\
& =\left(\left(\mathbf{D}-\lambda_{\text {mean }} \mathbf{I}\right)^{T}\left(\mathbf{D}-\lambda_{\text {mean }} \mathbf{I}\right)\right) / \lambda_{\text {mean }}^{2}
\end{aligned}
$$

which was suggested to us by Peter Basser [Basser 2000]. Note that the computation is efficient and does not require the computation of the eigenvalues. The measure is used to identify regions where $\lambda_{1}>>\lambda_{2} \geq \lambda_{3}$ and can be used to detect nerve fiber tracts.

Additional measures have been proposed by Westin et al. [Westin et al. 1997]. The authors define a linear isotropy $c_{l}$, a planar isotropy $c_{p}$, and an isotropy $c_{s}$ as

$$
\begin{aligned}
c_{l} & =\frac{\lambda_{1}-\lambda_{2}}{\lambda_{1}+\lambda_{2}+\lambda_{3}} \\
c_{p} & =\frac{2\left(\lambda_{2}-\lambda_{3}\right)}{\lambda_{1}+\lambda_{2}+\lambda_{3}} \\
c_{s} & =\frac{3 \lambda_{3}}{\lambda_{1}+\lambda_{2}+\lambda_{3}}
\end{aligned}
$$

The measures fall in the range $[0,1]$ and sum up to 1 and define therefore a barycentric space of anisotropies.

\subsection{Medical Applications}

DTI imaging has been successfully applied to diagnose various diseases. For stroke victims it has been shown that diffusion reduces in the demarcated ischemic region within minutes whereas changes in conventional $T_{2}$ weighted MRI images become apparent only after about three hours [Warach et al. 1992]. Assaf et al. report that DTI images are also sensitive to the pathophysiological state of white matter in brains diagnosed with Multiple Sclerosis [Assaf et al. 2002]. Zhang et al. note that the white matter regions adjacent to the edema surrounding a metastasis are characterized by heterogeneity in the diffusion anisotropy [Zhang et al. 2002]. Barnea-Goraly et al. show that regionally specific alterations of white matter integrity occur in patients with Fragile X Syndrome, a common form of hereditary mental retardation [Barnea-Goraly et al. 2003]. DTI has also been used to investigate the development of white matter tracts in adolescents and adults [Li and Noseworthy 2002].

The above examples demonstrate the importance of diffusion tensor imaging for medical diagnosis and research. It is important to note that, since the resolution of DTI is limited, small fibers adjacent to each other and branching fibers cannot be distinguished. Recent research attempts to improve the standard diffusion tensor model by using high angular resolution diffusion weighted acquisition [Ozarsland et al. 2001].

\section{Previous Work}

Diffusion tensor fields in the medical field have been originally visualized in two dimensions by representing a derived scalar measure over a data slice with a color map or a gray scale map [Pierpaoli et al. 1996]. Subsequent research has examined the visualization of directional tensor information over 2D slices via color mapping [Jones et al. 1997; Pierpaoli 1997]. Peled et al. visualize a slice of a diffusion tensor data set by indicating the in-plane component of the principal diffusion with a blue line and the out-of-plane components by colors ranging from green through yellow to red [Peled et al. 1998].

An interesting visualization of DTI slice images has been developed by Laidlaw et al. who use concepts from oil painting [Laidlaw et al. 1998]. The projection of the principal diffusion direction onto the image plane is encoded by the stroke direction and the out-of-plane component by the saturation of the red color component. Diffusion anisotropy is represented by the length/width ratio and transparency of brush strokes and the magnitude of the diffusion rate by the stroke texture frequency. Additional information is given using the lightness of the under-painting and an underlying checkerboard spacing.

More recently researchers have concentrated their efforts onto the $3 \mathrm{D}$ reconstruction and visualization of nerve fiber tracts. Xue et al. track nerve fibers by propagating a line from the center of a voxel along the direction of the maximum eigenvector until it exits the voxel [Xue et al. 1999]. Poupon et al. [Poupon et al. 1998] track white matter fibers using a Markovian model and the assumption that fiber tracts can not end in white matter. In a later paper the authors use knowledge of the low curvature of most fascicles and track them using a bending-energy minimizing scheme [Poupon et al. 2000]. In a previous paper we showed that fiber tracts can be visualized with streamtubes and hyperstreamlines [Wünsche and Lobb 2001].

Kindlmann et al. [Kindlmann and Weinstein 1999; Kindlmann et al. 2000] visualize the 3D geometry of the diffusion tensor field using a direct volume rendering technique with the color, lighting and opacity assignment governed by the underlying tensor field. Colors are determined by transforming a constant input vector with the tensor and by using the result to index a Hue-ball which is a $2 \mathrm{D}$ spherical color map. The hue of the resulting color reflects the principal diffusion direction and the saturation the diffusion anisotropy. Fiber tracts show up as regions of slowly varying saturated color.

\section{Visualizations}

\subsection{Color Mapped Surfaces}

Traditionally MRI data is displayed as a set of slices parallel to one of the coordinate planes. The optimal presentation of MRI slice images for improved perception of information has been researched by [van der Heyden et al. 1998].

As a basic example for color mapping consider figure 1 which shows nine equidistant horizontal slices through the brain from top 


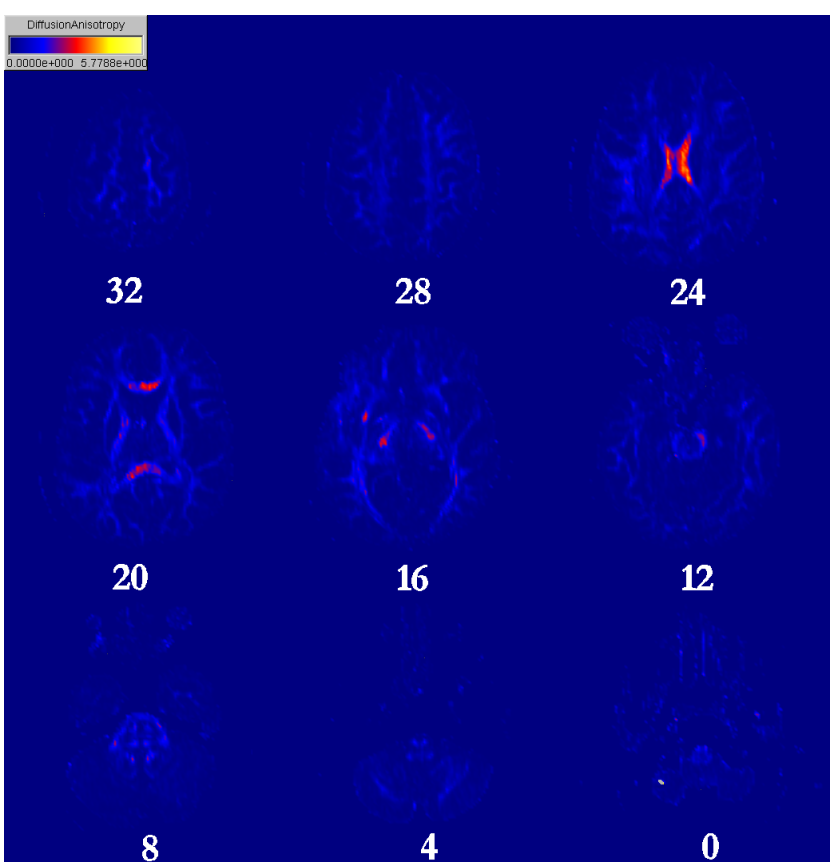

Figure 1: Horizontal slices (with numbers) color mapped with the diffusion anisotropy.

to bottom color mapped with the diffusion anisotropy $\lambda$ defined by equation 4 . The images are ordered from left to right and top to bottom.

White matter is indicated by saturated blue with the corpus callosum and some parts of the internal capsule colored red and yellow. Both gray matter and CSF have a very low anisotropy and are hence colored in black-blue.

It can be seen that naive use of color mapping does not differentiate anatomical structures from the background which makes the interpretation and analysis of the visualization difficult. For the remainder of this paper we classify background voxels by identifying an appropriate cut-off value from the histogram of the mean diffusivity of the image data set.

Fine structures and symmetries can be revealed by using cyclical color maps. Figure 2 (a) visualizes the mean diffusivity. Note that the contour density and contour normal direction of the resulting surface texture indicate the magnitude and direction of the mean diffusivity. The regions with a high mean diffusion gradient (high contour density) visible in the center and the periphery of the brain indicate fluid filled compartments.

Part (b) of the figure visualizes the diffusion anisotropy. Comparing the isocontours in white matter regions in the left and right brain hemisphere it can be seen that the anisotropy is almost symmetric in the anterior side of the brain but is slightly asymmetric in the posterior side with higher values for the left hemisphere. This result is in contrast to findings by [Peled et al. 1998] and could indicate that the white matter regions in the left hemisphere are more compact and therefore the fibers are more aligned [Faull 2000]. This hypothesis is consistent with the fact that the left hemisphere contains an additional brain region responsible for verbal abilities.

We believe that cyclical color maps as demonstrated above might be especially useful when examining degenerative white matter diseases [Lim et al. 1999; Highley et al. 1999; Thompson et al. 2001].

Regions of gray matter, white matter, and CSF can be displayed simultaneously using the segmentation function

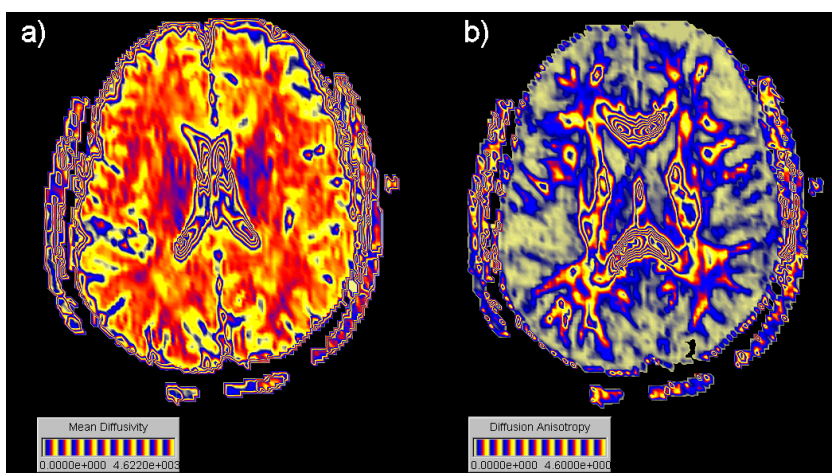

Figure 2: Horizontal slice (number 20) with the mean diffusivity (a) and the diffusion anisotropy (b) visualized using cyclical color maps (right).

$$
\lambda_{s}= \begin{cases}1 & \text { if } \lambda_{\text {aniso }} \geq 0.25 \text { and } 5 * 10^{-6}<\lambda_{\text {mean }}<10^{-3} \\ 2 & \text { if } \lambda_{\text {mean }} \geq 10^{-3} \\ 3 & \text { if } \lambda_{\text {aniso }}<0.25 \text { and } 5 * 10^{-6}<\lambda_{\text {mean }}<10^{-3} \\ 0 & \text { otherwise }\end{cases}
$$

The conditions for the values 1,2 , and 3 reflect the characterization of tissue types explained in section 2 and are chosen so that they indicate white matter, CSF and gray matter, respectively. The values are similar to the ones suggested by Pierpaoli et al. [Pierpaoli et al. 1996]. Figure 3 (a) shows the resulting segmentation using the colors red, green and blue, respectively. In contrast to the previous images this image allows the identification of the thalamus as the two blue regions between the lateral ventricle in green and the internal capsule in red.

Regions of predominant linear anisotropic, planar anisotropic and isotropic diffusion are identified by using a barycentric color map which visualizes the measures defined by equations 4-6.

A barycentric color map is constructed by assigning three different colors to the vertices of a triangle and by interpolating these colors for each point $P$ inside the triangle $\triangle A B C$ using the barycentric coordinates

$$
\alpha=\frac{\operatorname{area}(\triangle P B C)}{\operatorname{area}(\triangle A B C)}, \quad \beta=\frac{\operatorname{area}(\triangle P C A)}{\operatorname{area}(\triangle A B C)}, \gamma=\frac{\operatorname{area}(\triangle P A B)}{\operatorname{area}(\triangle A B C)}
$$

The barycentric coordinates define the weights of a convex sum of the triangle vertices which is equal to $P$, i.e., $\alpha A+\beta B+\gamma C=P$ where $0 \leq \alpha, \beta, \gamma \leq 1$ and $\alpha+\beta+\gamma=1$. The measures defined by equations 4-6 define a barycentric space of anisotropies and therefore can be visualized by mapping them onto the barycentric color map.

Figure 3 (b) indicates that the diffusion is predominantly linear in the genu (1) and splenium (2) of the corpus callosum and in the internal capsule (3), and more planar in the optic radiation (4) and in the more peripheral white matter regions. A higher linear anisotropy indicates a higher alignment of nerve fibers.

\subsection{Anatomical Landmarks}

The identification of anatomical structures can be facilitated by inserting easily recognizable features, so-called anatomical landmarks, into the 3D visualization. Two suitable structure are the ventricles and the eyes shown in figure 4 as smoothly shaded red and green isosurfaces, respectively. 


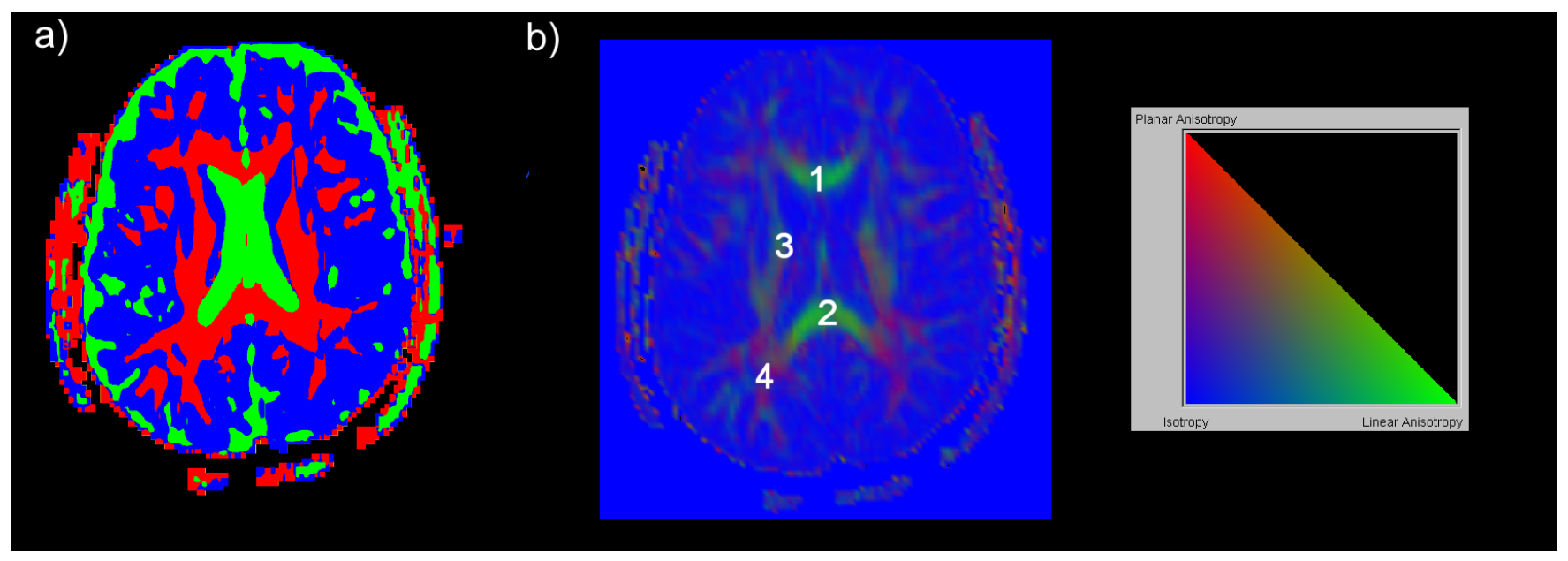

Figure 3: Horizontal slice (number 20) colored using a segmentation function (a) and a barycentric color map (b). In (a) red, green and blue indicate white matter, CSF and gray matter, respectively.

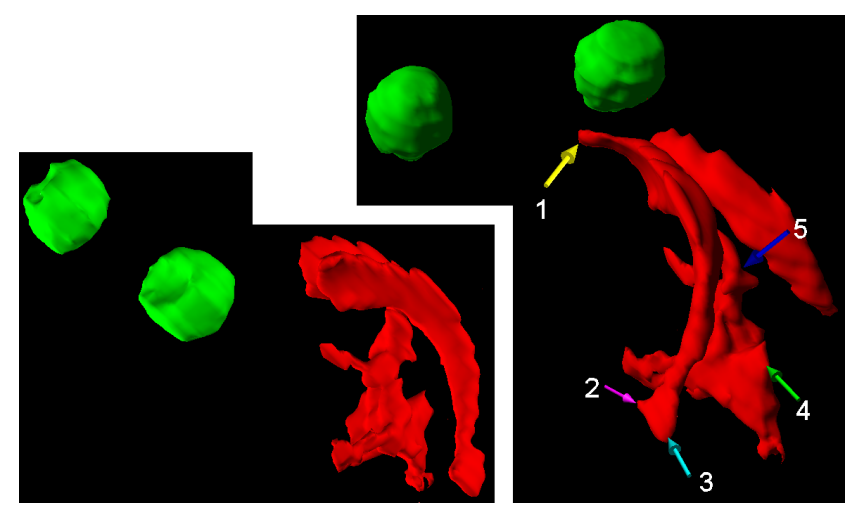

Figure 4: The eye balls (green) and the ventricles (red) represented as isosurfaces of the mean diffusivity. (1) anterior horn, (2) posterior horn and (3) inferior horn of the lateral ventricle, (4) fourth ventricle and (5) third ventricle.

The ventricles are approximated by the $1700 * 10^{-6} \mathrm{~mm} / \mathrm{s}$ isosurface of the mean diffusivity. Since the brain is surrounded by CSF, isosurfaces are also computed for fluid filled fissures and sulci between the brain and the skull and the ventricles must be extracted using a postprocessing step [Wünsche 2003a].

The eye balls are filled with a clear jelly (aqueous humor) and are consequently characterized by a very high isotropic diffusivity; in our case we use the $3500 * 10^{-6} \mathrm{~mm} / \mathrm{s}$-isosurface of the mean diffusivity. The cavity in the front of each eye ball indicates the position of the lens. The dimensions of the left eye ball are $23.8 \mathrm{~mm} \times 24.4 \mathrm{~mm} \times 23 \mathrm{~mm}$ which corresponds well with results reported in the literature (an average diameter of $24 \mathrm{~mm}$ and an average axial length of $25 \mathrm{~mm}$ including the cornea) [Wandell $\mathrm{n}$. d.]

Note that planar sections of the brain, such as the horizontal plane bisecting the eyes and the vertical plane dividing the brain hemispheres, also show the eyes and the ventricles. However, color mapped slices do not show the full 3D geometry of anatomical structures, they occlude one half of the brain and they do not aid the depth perception as is the case when using a shaded 3D structures such as the ventricles.

\subsection{Introducing Local Tensor Information}

\subsubsection{Ellipsoid-based Textures}

Informative textures can be created by color mapping diffusion ellipsoids with directional information and/or anisotropy measures. Textures are perceptually characterized by the spatial frequency, contrast and orientation of texture components [Schiffman 1996; Ware and Knight 1995]. In order to make use of the pattern recognition capabilities of the human visual system we use a dense distribution of color mapped diffusion ellipsoids and scale them such that they overlap in regions of high mean diffusivities. As a result regions of CSF exhibit an irregular pattern consisting of the visible sections of overlapping ellipsoids, white matter regions are characterized by regularly arranged long cigar shaped ellipsoids aligned along the nerve fiber tracts, and gray matter regions are represented by small approximately spherical ellipsoids. We encode additional information and further differentiate the textural appearance of regions with different tissue type by color mapping the ellipsoids.

White matter regions and the direction of nerve fiber tracts are emphasized by using the spherical color map shown in figure 5 (a). The hue, saturation and brightness of the color spectrum vary along the circumferential, longitudinal and radial directions of the sphere, respectively. We encode the direction of the maximum diffusivity by computing its spherical coordinates and by associating them with the hue and saturation of the color map. The diffusion anisotropy $\lambda_{\text {anisotropy }}$ is mapped onto the brightness parameter of the color spectrum.

The results of applying this color map to an ellipsoid-based texture are illustrated in figure 5. The visualization represents a horizontal slice through the brain. The color mapped ellipsoids are illuminated using ambient, diffuse and specular illumination (b) and ambient and diffuse illumination (c). The images on the right show a detail view of the images on the left.

It can be seen that specular illumination makes it difficult to differentiate tissue types since specular highlights dominate the image and obscure the ellipsoid color and the texture pattern generated by overlapping/non-overlapping ellipsoids. Using ambient illumination only results in the best differentiation between tissue types: gray matter appears black, CSF appears as regions with large overlapping darkly colored ellipsoids and white matter is represented by elongated ellipsoids with a highly saturated color. However, using ambient illumination only makes it difficult to perceive the $3 \mathrm{D}$ 

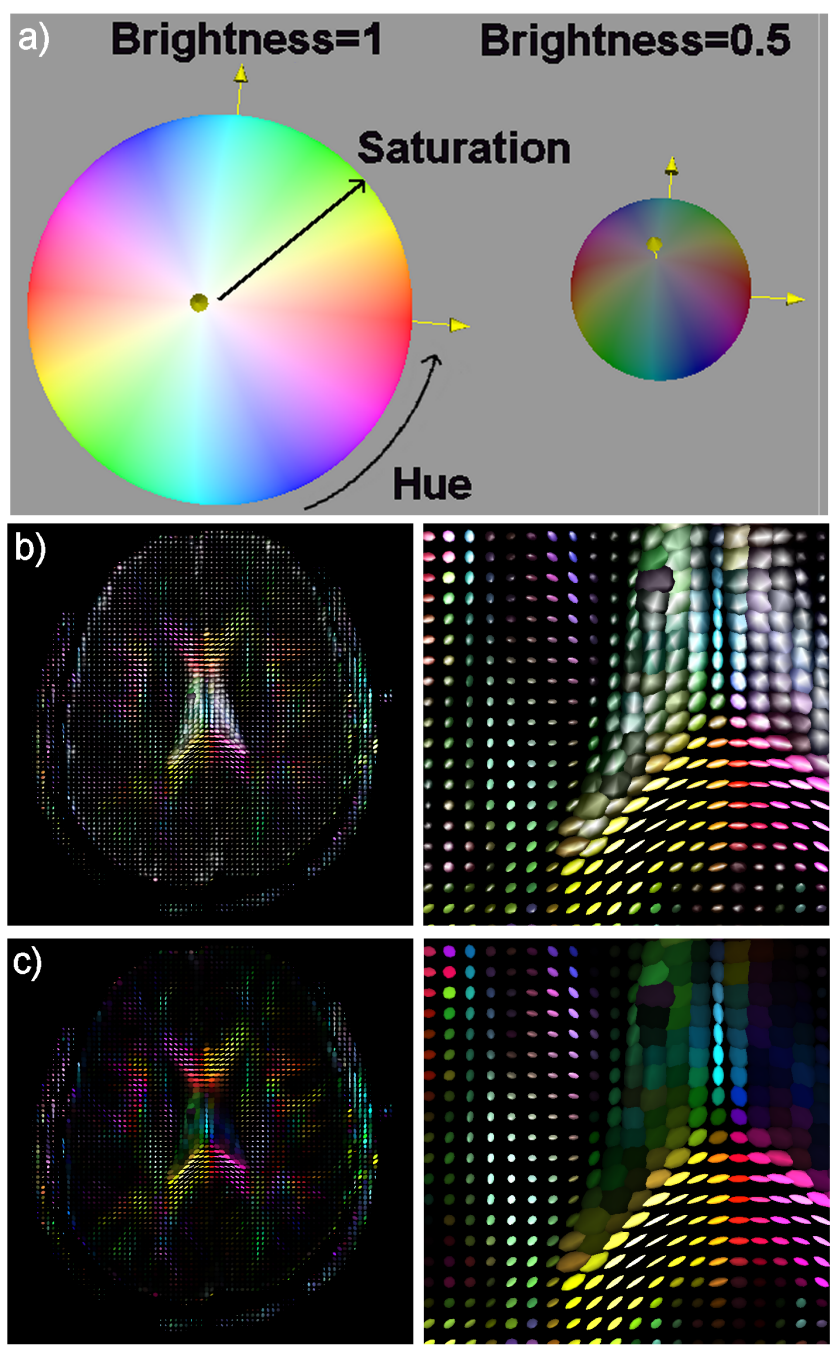

Figure 5: (a) A spherical color map with hue, saturation and brightness varying along the circumferential, longitudinal and radial direction of the sphere, respectively. The illustration shows the surfaces of the color map formed by choosing a constant brightness parameter of 1.0 (left) and 0.5 (right).

The fiber tract direction over a horizontal slice through the brain is visualized with an ellipsoid-based texture based on the above color map and by illuminating it using ambient, diffuse and specular illumination (b) or ambient and diffuse illumination (c). The images on the right show an enlargement of the region containing the splenium of the corpus callosum. shape of individual ellipsoids. Using ambient and diffuse illumination as in (c) combines both good shape perception and good differentiation of tissue types. Alternative ellipsoid based textures are suggested in [Wünsche 2004].

\subsubsection{Anisotropy Modulated Line Integral Convolution}

Disadvantages of the ellipsoid-based textures are that ellipsoids visualize the diffusion direction and hence the nerve fiber direction only at individual points, that the 3D shape of ellipsoids can be hard to perceive, and that there is little perceptual continuity between ellipsoids aligned along a nerve fiber tract. As an improved method for visualizing the fiber direction in DTI slice images we propose Anisotropy Modulated Line Integral Convolution (AMLIC). A 2D AMLIC texture can be described in simple words as a blend of a Line Integral Convolution (LIC) texture of the maximum diffusion direction with the color mapped mean diffusivity.

Line Integral Convolution (LIC), originally proposed by Cabral and Leedom [Cabral and Leedom 1993], is an effective method to visualize vector fields by using curvilinear filters to locally blur an input noise texture $I$ along a vector field $\mathbf{v}$.

An AMLIC texture is created by first computing the LIC texture from the maximum diffusion direction. As explained in the introduction nerve fiber tracts are characterized by a relatively high and anisotropic diffusion in the fiber direction. It is therefore possible to extract nerve fiber tracts as streamlines by integrating in the direction of the maximum diffusivity in regions of high anisotropy [Basser 2000]. The LIC texture is defined by convolving a white noise texture with the maximum diffusion direction. This is achieved by tracing through the center of each output texture pixel a short streamline along the maximum diffusion direction projected onto the image plane. The intensity of the output texture pixel is the sum of the intensities of the input texture pixels along that streamline weighted with the integral of the convolution kernel which in our case is a simple box filter. The length of the convolution kernel at a pixel is proportional to the angle between the maximum diffusion direction and the textured surface. Since anisotropic diffusion can also be registered due to noise or eddy currents in fluid filled compartments the maximum diffusivity at any step during the streamline integration must exceed a certain predefined limit and the streamline must exceed a specified minimum length and must be sufficiently smooth.

The LIC texture is color mapped with the diffusion anisotropy and for each pixel a blending factor monotonically increasing with the diffusion anisotropy is defined. We obtained good results by using a power function with the exponent 0.2. More details are found in [Wünsche 2003a]. The LIC texture is blended with the color mapped mean diffusivity using the OpenGL "GL_BLEND" operation [Woo et al. 1997]. The texture color of pixel $(i, j)$ is then given by

$O_{i j}=\alpha_{i j} C_{i j}+\left(1-\alpha_{i j}\right) D_{i j} / /$ the output texture color

where

$$
\begin{gathered}
C_{i j}=L_{i j} c\left(\lambda_{\text {aniso }}(i, j)\right) \quad / / \text { the LIC texture } \\
\text { // color mapped with the diffusion } \\
\text { // anisotropy at pixel centers } \\
\text { // the LIC texture obtained from the } \\
\text { // principal diffusion direction } \\
L_{i j} \quad / / \text { a texture representing } \\
D_{i j}=d\left(\lambda_{\text {mean }}(i, j)\right) \text { // the color mapped mean diffusivity } \\
\text { // }(t), d(t) \quad / / \text { color spectra indexed with } t \in\left[t_{\text {min }}, t_{\text {max }}\right] \\
\alpha_{i j}=\left(\lambda_{\text {aniso }}(i, j)\right)^{0.2} / / \text { a blending factor } \\
\text { // monotonically increasing with } \\
\text { // the diffusion anisotropy }
\end{gathered}
$$



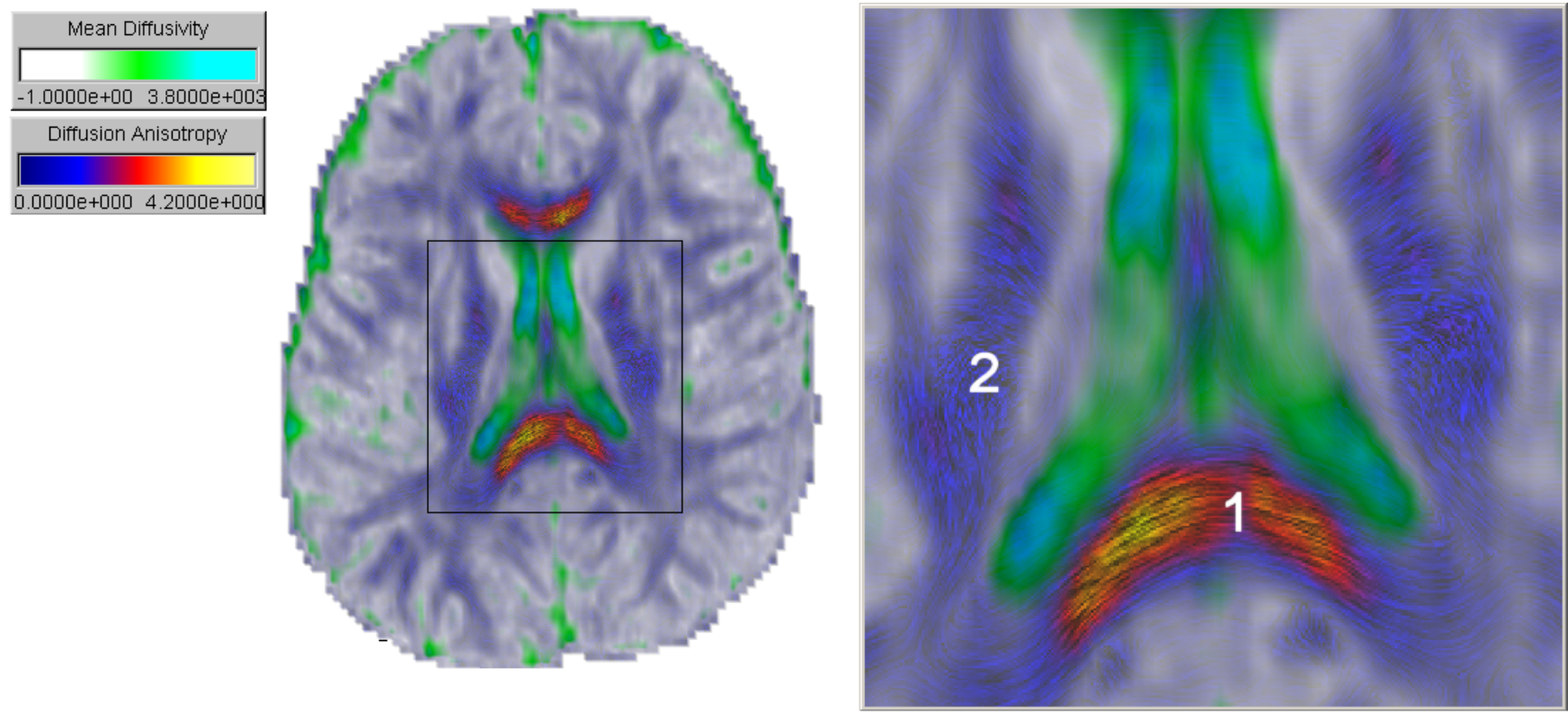

Figure 6: Fiber tract direction over a horizontal slice through the brain visualized using anisotropy modulated line integral convolution (AMLIC). The nerve fibers in the splenium of the corpus callosum (1) are parallel to the image plane whereas nerve fibers in the posterior limb of the internal capsule (2) are almost vertical to it.

The resulting texture has three properties: regions of high anisotropy feature a LIC like texture which indicates the 3D nerve fiber direction. Long texture elements indicate nerve fibers tangential to the textured surface whereas very short point-like texture elements indicate nerve fibers almost orthogonal to the textured surface. Regions which are not textured have a low anisotropy and therefore represent either gray matter or cerebral spinal fluid. If an appropriate color map is chosen (we found that three equally distributed colors over the range of scalar values of the mean diffusivity work well) then gray matter is indicated by the color(s) associated with low values of the mean diffusivity and CSF is indicated by the color(s) associated with high values of the mean diffusivity.

Note that by blending the LIC texture and the color mapped mean diffusivity we take into account the fact that there is no clearcut boundary between white matter and gray matter. The resulting visualization therefore has advantages over image segmentation methods.

An example is given in figure 6 . The cyan and green regions represent areas of high mean diffusivity and low anisotropy and therefore indicate fluid filled compartments. The whitish regions represent areas of low anisotropy and low mean diffusivity and therefore indicate gray matter. Textured regions exhibit a high anisotropy and indicate white matter. Very long texture components indicate fiber tracts parallel to the image plane, e.g., in the splenium of the corpus callosum (1). In contrast a noise-like texture with very short texture components indicates fiber tracts almost orthogonal to the image plane, e.g., in the posterior limb of the internal capsule (2).

\subsection{Extracting Nerve Fiber Tracts}

\subsubsection{Streamlines \& Streamtubes}

As explained previously nerve fiber tracts are characterized by a relatively high and anisotropic diffusion in the fiber direction. It is therefore possible to extract nerve fiber tracts as streamlines by integrating in the direction of the maximum diffusivity in regions of high anisotropy. Since similar diffusion properties can also be reg- istered due to noise or eddy currents in fluid filled compartments the anisotropy and maximum diffusivity at any step during the streamline integration must exceed certain predefined limits (we call this requirement the "integration condition") and the streamline must exceed a specified minimum length.

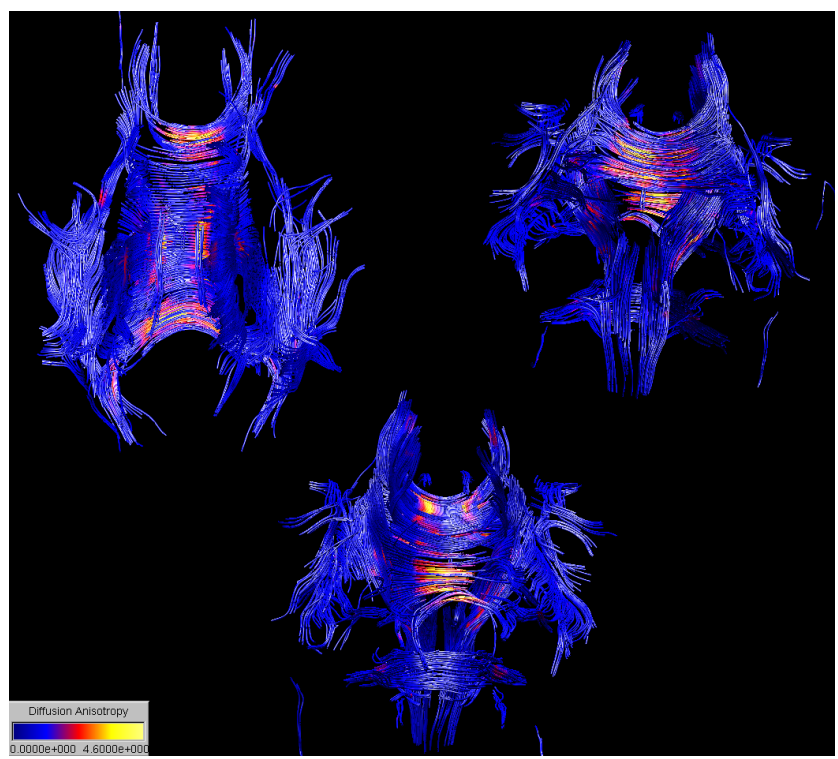

Figure 7: Fiber tracts visualized using streamtubes color mapped with the diffusion anisotropy. The image shows in clockwise direction starting from the left the top, front and back side of the brain.

Representing the tracked fibers by simple streamlines results in images where the exact 3D geometry is difficult to understand since depth cues due to shading and overlay are missing. A vastly im- 
proved visualization is obtained by using streamtubes which are formed by fitting a cylinder with constant radius around the streamline. Figure 7 shows an example which was generated by using the start point condition $\lambda_{\text {anisotropy }} \geq 0.7$ and $\lambda_{1} \geq 1000$ and the integration condition $\lambda_{\text {anisotropy }} \geq 0.3$ and $\lambda_{1} \geq 750$. In order to obtain a dense image $2 \times 2 \times 2$ start points per grid cell of the DTI data set were chosen. Visual cluttering was avoided by limiting the maximum number of streamtubes intersecting a grid cell to eight. The step size used was $0.75 \mathrm{~mm}$ and the minimum streamline length was $35 \mathrm{~mm}$. Subsubsection 4.4 .3 contains a discussion of the choice of parameters.

\subsubsection{Hyperstreamlines}

Streamlines and streamtubes contain only information about the major diffusion direction. More information can be visualized by using hyperstreamlines whose cross section represents the transverse diffusion in fiber tracts [Wünsche and Lobb 2001]. Note that it's not necessary to normalize the hyperstreamlines such that the maximum diameter is constant as done in [Zhang et al. 2003].

Figure 8 depicts the nerve fiber tracts in the brain visualized with hyperstreamlines. Perception of the $3 \mathrm{D}$ geometry is improved by inserting the eyes and the ventricles as anatomical landmarks as explained in subsection 4.2. The images were generated using one start point per grid cell of the DTI data set with a start point condition of $\lambda_{\text {anisotropy }} \geq 0.7$ and $\lambda_{1} \geq 1000$ and an integration condition of $\lambda_{\text {anisotropy }} \geq 0.3$ and $\lambda_{1} \geq 750$. Only one streamline per grid cell of the DTI data set was allowed and a step size of $0.75 \mathrm{~mm}$ and a minimum length of $35 \mathrm{~mm}$ were used for the computation. The hyperstreamlines are color mapped with the maximum diffusivity.

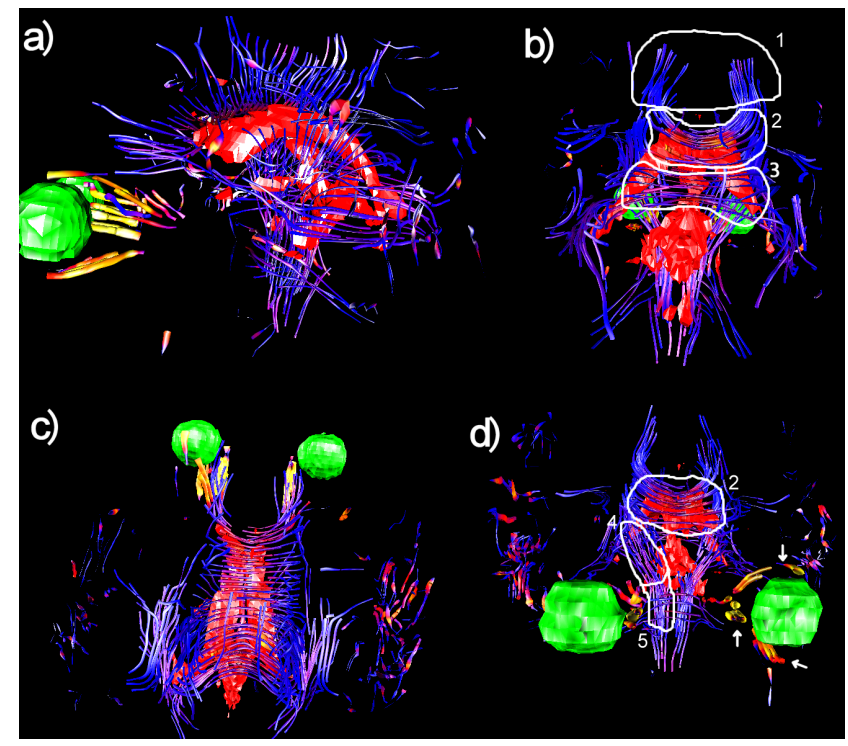

Figure 8: Nerve fiber tracts visualized by hyperstreamlines color mapped with the maximum diffusivity: 1-Corona radiata, 2-Corpus callosum, 3-Splenium of the corpus callosum, 4-Internal capsule, 5-Cerebral peduncles. The images shows the left (a), back (b), top (c) and front (d) side of the brain.

Comparing the visualization with photographs and drawings from the literature [England and Wakely 1991; Guyton 1987; Hanaway et al. 1998] makes it possible to identify the main fiber tracts as indicated in the image. The results were verified by consulting a neuroanatomist.
Close to the eye balls three groups of hyperstreamlines can be differentiated and are indicated in figure 8 (d) by arrows. The arrow on the left denotes the optic nerve whereas the top and bottom arrow indicate the opthalmic division and the maxilliary division of the trigeminal nerve (cranial nerve $\mathrm{V}$ ), which conducts sensory impulses from the cornea and the skin. The oculomotor nerve, which controls the eye muscle, is not visible presumably because of its relatively small diameter. The location of the optic nerve is more inward than expected from the literature [Guyton 1987, p.41].

It is interesting to note that the image shows groups of hyperstreamlines for each nerve even though the nerves have a relatively small diameter. This is most likely due to branching of the nerves and the presence of the ciliary ganglion. Note that the maximum diffusivity indicated by the color mapping is considerably higher in the eye region than in all other white matter regions except for some parts of the corpus callosum.

In order to better differentiate features stricter conditions for the definition of hyperstreamlines can be used which results in fewer streamlines concentrated along the major nerve fiber tracts. Figure 9 shows a posterior, superior, and posterior left lateral view of a visualization obtained by using the same visualization parameters as in the previous image except that the starting condition for streamline integration was changed to $\lambda_{\text {anisotropy }} \geq 1.0$ and $\lambda_{1} \geq 1000$. The hyperstreamlines are color mapped with the diffusion anisotropy.

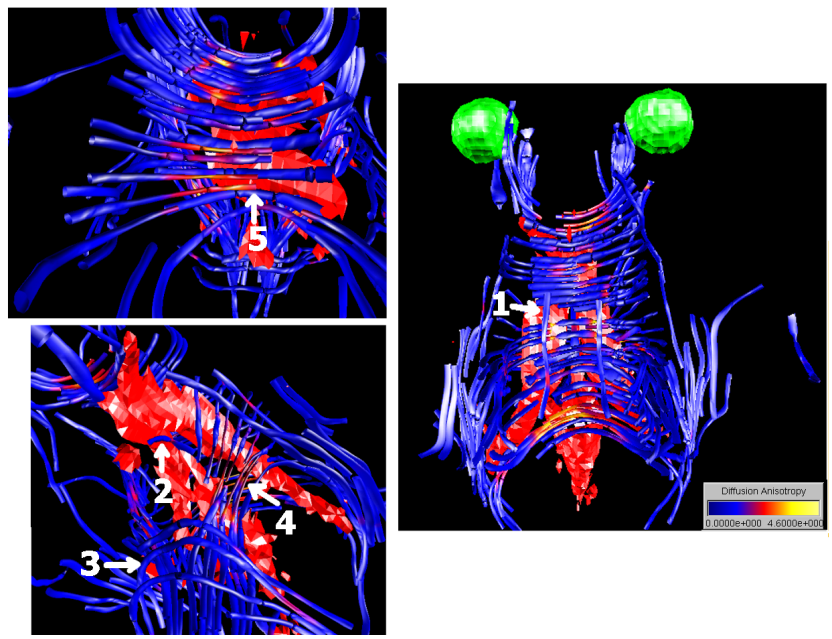

Figure 9: Close up views of a visualization of fiber tracts using hyperstreamlines color mapped with the diffusion anisotropy: 1cingulum, 2-fornix, 3-middle cerebellar peduncle, 4-posterior limb of the internal capsule, 5-splenium of the corpus callosum.

The image indicates an approximately cylindrical anisotropy in most regions. Noticeable exceptions are the posterior limb of the internal capsule (4) and the splenium of the corpus callosum (5) where the minimum diffusivity is significantly reduced and the maximum diffusivity is increased which yields a high diffusion anisotropy.

Several interesting features can be identified from the figure. The hyperstreamline indicated by (2) inferior to the corpus callosum and bending downward is the fornix which takes part in the integrative function of the brain and terminates in the hippocampus. Superior to the corpus callosum is the cingulum (1) which connects the cingular jarus with the temporal lobe and the hippocampus. The pons is identified by the presence of the middle cerebellar peduncle (3) which wraps around the pyramidal tract.

The color coding of the hyperstreamlines in figure 8 and 9 indi- 
cates that the mean diffusivity and the diffusion anisotropy usually don't vary much even though the packing density of nerve fibers in different white matter regions can vary by up to a factor of five $\left(60000-70000 / \mathrm{mm}^{2}\right.$ in the pyramidal tract and $338000 / \mathrm{mm}^{2}$ in the corpus callosum [Pierpaoli et al. 1996]).

\subsubsection{Influence of parameters}

Choosing the parameters for the streamline computation involves a compromise between the amount of information displayed, visual cluttering and the amount of noise and artifacts in the image. As mentioned previously noise and artifacts due to eddy currents can be minimized by specifying a suitable minimum length for the streamlines. Care must be taken that the specified value is not too high since otherwise short fiber tracts such as the ones in the vicinity of the eye are eliminated. We found that a minimum length of $35 \mathrm{~mm}$ removed most of the short hyperstreamlines in the peripheral regions of the brain but had no noticeable influence on the anatomical features identified. Figure 10 shows two visualizations generated with a minimum length of $23 \mathrm{~mm}$ (a) and $35 \mathrm{~mm}$ (b).

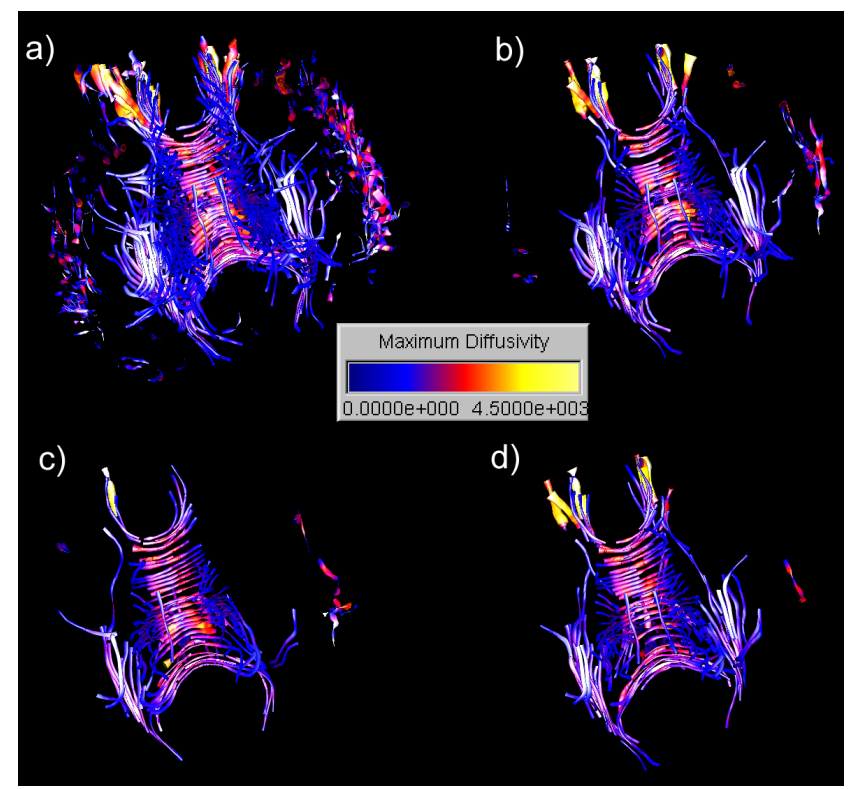

Figure 10: Hyperstreamlines generated using the parameters in table 1.

Other important parameters are the choice of the start points for the computation and the integration condition along the trajectory of the hyperstreamline.

In the previously presented images the start points for fiber tracking were the centers of all grid cells of the DTI image data set which fulfill a given start point condition. The start point condition has to guarantee that streamlines are only generated inside of fiber tracts and we therefore specify both a minimum anisotropy and a minimum maximum diffusivity. The second restriction is necessary to eliminate "anisotropic noise". The integration condition must guarantee that the streamline stays inside the fiber tract but has to allow for fluctuations in the anisotropy and maximum diffusivity due to noise. Consistency is best maintained by defining the integration condition as a weak form of the start point condition. Making the integration condition too strong results in streamlines with multiple gaps which often do not fulfill the minimum length criteria. Too weak a condition, on the other hand, generates few, if any, additional fiber tracts, but rather leads to visual cluttering.
Figure 10 shows as an example the images generated with the parameter choices in table 1 . The hyperstreamlines are color mapped with the maximum diffusivity. Part (b) and (c) of the figure demonstrate that increasing the minimum anisotropy at the start point from 0.6 to 1.0 removes the trigeminal nerve, most of the superior longitudinal fasciculus and the cingulum and optic nerve in the right hemisphere. Part (d) of the figure demonstrates that increasing the anisotropy instead to 0.7 and introducing additionally a minimum value for the maximum diffusivity maintains all previously identified fiber tracts but removes artifacts in the peripheral brain regions not eliminated by the minimum length condition. The nerve fiber tracts in the vicinity of the eye are most sensitive to variations of the start point and integration condition.

Note that we do not use a curvature criterion for tracking nerve fiber tracts as done in [Poupon et al. 2000]. Our examples demonstrate that this is not necessary and the restriction might be counterproductive when visualizing diseased or abnormal neuroanatomy.

\begin{tabular}{|c|c|c|c|}
\cline { 2 - 4 } \multicolumn{1}{c|}{} & Start point condition & Integration condition & min. length \\
\hline a) & $\lambda_{\text {anisotropy }} \geq 0.6$ & $\lambda_{\text {anisotropy }} \geq 0.2$ & $23 \mathrm{~mm}$ \\
\hline b) & $\lambda_{\text {anisotropy }} \geq 0.6$ & $\lambda_{\text {anisotropy }} \geq 0.3$ & $35 \mathrm{~mm}$ \\
\hline c) & $\lambda_{\text {anisotropy }} \geq 1.0$ & $\lambda_{\text {anisotropy }} \geq 0.3$ & $35 \mathrm{~mm}$ \\
\hline d) & $\lambda_{\text {anisotropy }} \geq 0.7$ and \\
& $\lambda_{1} \geq 1000.0$ & $\lambda_{\text {anisotropy }} \geq 0.3$ and & $35 \mathrm{~mm}$ \\
$\lambda_{1} \geq 1000.0$ & \\
\hline
\end{tabular}

Table 1: Parameter choices for the images in figure 10.

\section{Conclusion}

Visualizing the diffusion tensor field in the brain gives an in vivo view of anatomical structures which was previously unobtainable. Using an incremental approach starting with color mapped slices and extending to $2 \frac{1}{2} \mathrm{D}$ and $3 \mathrm{D}$ techniques diffusion data can be systematically explored.

New insight into diffusion tensor data is gained using barycentric color maps which show the distribution of anisotropies over a region and indicate possible fiber tract crossings. Cyclical color maps reveal structure in scalar fields and by using them we found an interesting asymmetry in the diffusion anisotropy.

Ellipsoid-based textures provide a visualization of fiber direction and tissue types or anisotropy properties. A high amount of information can be encoded into slice images using anisotropy modulated line integral convolution. The technique does not only indicate three dimensional fiber direction but also provides a visual segmentation of tissue types.

Using streamtubes and hyperstreamlines makes it possible to obtain high quality $3 \mathrm{D}$ visualizations of the nerve fiber structure. We were able to demonstrate that the major principal pathways in the brain can be extracted and readily identified. Care must be taken when choosing the appropriate visualization parameters.

In contrast to Zhang et al. [Zhang et al. 2003] we do not normalize the transverse diffusivities encoded in hyperstreamlines and therefore we represent additional information. We also employ different methods for the selection of streamlines and we perform the fiber tracking using a simple, fast and flexible general purpose streamline tracking algorithm implemented within our visualization toolkit [Wünsche 2003b; Wünsche 2003a].

The understanding of the complicated geometry of a fiber tract visualization can be improved by inserting anatomical landmarks. We listed several examples of landmarks and showed how they can be generated interactively with our visualization toolkit. 


\section{Future Research}

In future we intend to use our visualization toolkit for the exploration of various white matter diseases. Current research indicates that DTI is superior to traditional MRI imaging and using our advanced visualization methods might help to better understand the development of various white matter diseases and the anatomical abnormalities in neurological disorders such as schizophrenia. Ideally we would like to obtain a series of data sets taken over time which makes it possible to create an animated visualization of the progress of a neurodegenerative disease.

We are also interested in qualitatively comparing our general purpose streamline tracking algorithms with specialized methods based on Markov-chain models and diffusion-advection processes.

Finally we want to visualize simultaneously a DTI data set and the corresponding functional MRI (fMRI) data set. By using visualization methods similar to the one suggested by Worsley et al. [Worsley et al. 1996] it would be possible to simultaneously display anatomical and functional information. Functional MRI shows regions of activity in the brain due to the detection of metabolic processes characterized by a change in the blood oxygen level [ $\mathrm{Ny}-$ comed Amersham Ltd. 1999]. Improved very fast MR imaging sequences allow simultaneous fMRI and DTI [Aronen et al. 1999]. This technique would be especially helpful when understanding regenerative processes in the brain for which little is known about the relationship between anatomical and functional regeneration [Horner and Gage 2000].

Other functional brain imaging techniques have been presented that allow the measurement of electrophysiological, hemodynamic, and neurochemical processes that underlie normal and pathological brain function [Baillet et al. 2001]. Initial work on the integration of MRI, fMRI, and EEG and MEG source reconstruction techniques is presented in [Wagner and Fuchs 2001] and we would like to further progress this exciting research.

\section{Acknowledgments}

We would like to thank Peter J. Basser from the National Institute of Health, Bethesda, MD, for valuable discussions and Carlo Pierpaoli for providing us with the diffusion tensor data set of a healthy brain.

\section{References}

Aronen, H. J., Korvenoja, A., Martinkauppi, S., Perkiö, J., Karonen, J., AND CARlson, S. 1999. Clinical applications of functional magnetic resonance imaging. International Journal of Bioelectromagnetism 1, 1 (May), 2334. URL: http://www.ee.tut.fi/rgi/ijbem/volume1/ number1/html/ar5.htm.

Assaf, Y., Ben-Bashat, D., Chapman, J., Peled, S., Biton, I. E., Kafri, M., Segev, Y., Hendler, T., Korczyn, A. D., Graif, M., And Cohen, Y. 2002. High b-value qspace analyzed diffusion-weighted MRI: Application to multiple sclerosis. Magnetic Resonance in Medicine 47, 1 (Jan.), 115-126. URL: http://www.tau.ac.il/lifesci/brain/ mapunit/publication/115-126.pdf.

BAillet, S., Mosher, J. C., AND LeAhy, R. M. 2001. Electromagnetic brain mapping. IEEE Signal Processing Magazine 18, 6 (Nov.), 14-30.

Barnea-Goraly, N., Eliez, S., Hedehus, M., Menon, V., White, C. D., Moseley, M., And Reiss, A. L. 2003. White matter tract alterations in fragile $\mathrm{X}$ syndrome: Preliminary evidence from diffusion tensor imaging. American Journal of Medical Genetics (Part B: Neuropsychiatric Genetics) 118B, 1 (Apr.), 81-88. URL: http://spnl.stanford.edu/publications/ pdfs/Barnea_G_FX_DTI_(AJMG02).pdf.

BAsser, P. J., AND Pierpaoli, C. 1996. Microstructural and physiological features of tissues elucidated by quantitativediffusion-tensor MRI. Journal of Magnetic Resonance Series B 111, 3 (June), 209-219.

Basser, P. J., Mattiello, J., And Bihan, D. L. 1994. MR diffusion tensor spectroscopy and imaging. Biophysical Journal 66, 259-267.

BASSER, P. J. 1995. Inferring microstructural features and the physiological state of tissues from diffusion-weighted images. NMR in Biomedicine 8, 7-8, 333-344.

BASSER, P. J., 2000. Personal communication, May.

CABral, B., AND LeEdom, L. C. 1993. Imaging vector fields using line integral convolution. In Computer Graphics (SIGGRAPH '93 Proceedings), Addison Wesley, J. T. Kajiya, Ed., vol. 27, ACM SIGGRAPH, 263-272.

England, M. A., And Wakely, J. 1991. A Colour Atlas of the Brain \& Spinal Cord - An Introduction to Normal Neuroanatomy. Wolfe Publishing Ltd., 2-16 Torrington Place, London WCIE 7LT, England.

FAUlL, P. R., 2000. Personal communication, 28th September.

Guyton, A. C. 1987. Basic Neuroscience - Anatomy and Physiology. W.B. Saunders Company.

Hanaway, J., Woolsley, T. A., Gado, M. H., And Roberts, JR., M. P. 1998. The Brain Atlas. Fitzgerald Science Press, Bethesda, Maryland.

Highley, J. R., Esiri, M. M., McDonald, B., CortinaBorja, M., Herron, B. M., AND Crow, T. J. 1999. The size and fibre composition of the corpus callosum with respect to gender and schizophrenia: A post mortem study. Brain 122, 99 - 110. URL: http://www.psychiatry.ox.ac.uk/powic/ size_fibre_composition.html.

Horner, P. J., AND Gage, F. H. 2000. Regeneration of the damaged central nervous system. Nature 407, 6807 (Oct.), 963970 .

Jones, D. K., Williams, S. C. R., And Horsfield, M. A. 1997. Full representation of white-matter fibre direction on one map via diffusion tensor analysis. In Proc. 5th Int. Soc. of Mag. Res. in Med., 1743.

Kindlmann, G., And Weinstein, D. 1999. Hue-balls and lit-tensors for direct volume rendering of diffusion tensor fields. In Proceedings of Visualization '99, D. Ebert, M. Gross, and B. Hamann, Eds., IEEE, 183 - 189.

Kindlmann, G., Weinstein, D., And Hart, D. 2000. Strategies for direct volume rendering of diffusion tensor fields. IEEE Transactions on Visualization and Computer Graphics 6, 2 (Apr.), 124-138. URL: http://www.sci.utah.edu/ publications/gk_tvcg00/strategies-tensor.pdf.

Laidlaw, D. H., Ahrens, E. T., Kremers, D., And ReadHEAD, C. 1998. Visualizing diffusion tensor images of the mouse spinal cord. In Proceedings of Visualization '98, Computer Society Press, IEEE, 127 - 134. 
LI, T.-Q., And Noseworthy, M. D. 2002. Mapping the development of white matter tracts with diffusion tensor imaging. Developmental Science 5, 3, 293-300. URL: http://cogsci. ucsd.edu/DCNL/pdf/li.pdf.

Lim, K. O., Hedehus, M., Moseley, M., de Crespigny, A., Sullivan, E. V., And Pfefferbaum, A. 1999. Compromised white matter tract integrity in schizophrenia inferred from diffusion tensor imaging. Archives of General Psychiatry 56, 4 (Apr.), 367-374.

Nycomed Amersham Ltd., 1999. Nycomed Amersham intercontinental continuing education in radiology, course on functional imaging. 11-13 June, Oslo, Norway, URL: http://www . na-imaging.com/common/nicer/horiz99/0sloabs.htm.

Ozarsland, E., Vemuri, B., Silver, X., DeFord, M., And MARECI, T. 2001. Fiber tract mapping with magnetic resonance diffusion tensor imaging. National High Magnetic Field Laboratory Report 8, 3, 17-18. URL: http://www.magnet.fsu. edu/publications/reports/summer01screen.pdf.

Peled, S., Gudbjartsson, H., Westin, C.-F., KikiNIS, R., AND Jolesz, F. A. 1998. Magnetic resonance imaging shows orientation and asymmetry of white matter fiber tracts. Brain Research 780 (Jan.), 27 33. URL: http://splweb.bwh.harvard.edu:8000/pages/ papers/peled/htmlBrainRes.html.

Pierpaoli, C., Jezzard, P., Basser, P. J., Barnett, A., And CHIRo, G. D. 1996. Diffusion tensor MR imaging of the human brain. Radiology 201, 3 (Dec.), 637 - 648.

PIERPAOLI, C. 1997. Oh no! one more method for color mapping of fiber tract direction using diffusion MR imaging data. In Proc. 5th Int. Soc. of Mag. Res. in Med., 1741.

Poupon, C., Mangin, J.-F., Frouin, V., Régis, J., Poupon, F., Pachot-Clouard, M., Bihan, D. L., and Bloch, I. 1998. Regularization of MR diffusion tensor maps for tracking brain white matter bundles. In Medical Image Computing and Computer-Assisted Intervention - MICCAI'98, Springer Verlag, Cambridge, MA, W. M. Wells, A. Colchester, and S. Delp, Eds., Lecture Notes in Computer Science 1496, 489 - 498.

Poupon, C., Clark, C. A., Frouin, V., Régis, J., Bloch, I., Le Bihan, D., AND Mangin, J.-F. 2000. Regularization of diffusion-based direction maps for the tracking of brain white matter fascicles. Neuroimage 12, 2 (Aug.), 184-195.

SChiffMan, H. R. 1996. Sensation and Perception: An Integrated Approach, $4^{\text {th }}$ ed. John Wiley \& Sons.

Thompson, P. M., NARR, K. L., Blanton, R. E., AND TogA, A. W. 2001. The Corpus Callosum. Kluwer Academic Press, The Netherlands, ch. 6 - Mapping Structural Alterations of the Corpus Callosum during Brain Development and Degeneration. Eran Zaidel and Marco Iacoboni, editors, URL: http://www. Ioni.ucla.edu/ thompson/MarcoChpt/MarcoChpt_full.html.

van der Heyden, J. E., Carpendale, M. S. T., InKPen, K., AND ATKINS, M. S. 1998. Visual presentation of magnetic resonance images. In Proceedings of Visualization '98, Computer Society Press, D. Ebert, H. Hagen, and H. Rushmeier, Eds., IEEE, $423-426$.
WAGNER, M., AND FUCHS, M. 2001. Integration of functional MRI, structural MRI, EEG, and MEG. International Journal of Bioelectromagnetism 3, 1 (Mar.). URL: http://www.ee.tut. $\mathrm{fi} / \mathrm{rgi} / \mathrm{ijbem} / \mathrm{volume}$ /number1/pdf/wagner .pdf.

WANDELL, B. A. Useful numbers in vision science. URL: http: //white.stanford.edu/html/numbers/numbers.html.

Warach, S., Chien, D., Li, W., Ronthal, M., And EdelMAN, R. R. 1992. Fast magnetic resonance diffusion-weighted imaging of acute human stroke. Neurology 42, 9 (Sept.), 1717-1723. (published erratum appears in Neurology 1992 Nov;42(11):2192).

WARE, C., AND KNIGHT, W. 1995. Using visual texture for information display. ACM Transactions on Graphics 14, 1 (Jan.), 3-20.

Westin, C.-F., Peled, S., Gubjartsson, H., Kikinis, R., AND JOLESZ, F. A. 1997. Geometrical diffusion measures for MRI from tensor basis analysis. In Proceedings of ISMRM, 5th meeting. URL: http://www.spl.harvard.edu:8000/ pages/ppl/westin/papers/smr97/htmlversion.html.

Woo, M., NeIDer, J., AND DaVIS, T. 1997. OpenGL Programming Guide, $3^{r d}$ ed. Addison-Wesley Publication Company Inc.

Worsley, K. J., Marrett, S., Neelin, P., Vandal, A. C., Friston, K. J., AND Evans, A. C. 1996. A unified statistical approach for determining significant signals in images of cerebral activation. Human Brain Mapping 4, 58 - 73.

WÜNSCHE, B. C., AND LobB, R. 2001. The visualization of diffusion tensor fields in the brain. In Proceedings of METMBS'01, CSREA Press, 498 - 504

WÜNSCHE, B. C. 2003. A Toolkit for the Visualization of Tensor Fields in Biomedical Finite Element Models. PhD thesis, University of Auckland. (submitted for publication).

WÜNSCHE, B. C. 2003. A toolkit for visualizing biomedical data sets. In Proceedings of GRAPHITE 2003, ANZGRAPH and SEAGRAPH, 167-174.

WüNSCHE, B. C. 2004. Advanced texturing techniques for the effective visualization of neuroanatomy from diffusion tensor imaging data. In Proceedings of the Second Asia-Pacific Bioinformatics Conference (APBC '04). (accepted for publication).

Xue, R., van Zijl, P. C. M., Crain, B. J., Solaiyappan, M., AND MORI, S. 1999. In vivo three-dimensional reconstruction of rat brain axonal projections by diffusion tensor imaging. Magnetic Resonance in Medicine 42, 6 (Dec.), 1123-1127.

Zhang, S., Laidlaw, D. H., Bastin, M. E., Sinha, S., AND DEISBOECK, T. S. 2002. Application of DTI visualization and analysis on a data set from a brain tumor patient. In Proceedings of IEEE Visualization '02. Poster session, published on conference DVD, URL: http: //www.cs.brown.edu/research/ vis/lists/pdf/Zhang-2002-AOD.pdf.

Zhang, S., Demiralp, C., AND Laidlaw, D. H. 2003. Visualizing diffusion tensor MR images using streamtubes and streamsurfaces. IEEE Transactions on Visualization and Computer Graphics 9, 4 (Oct.), 454-462. 